\title{
Mathematical Modelling of Robust Optimization for Integer Programming Problem
}

\author{
Gyan Bahadur Thapa \\ Central Campus, Institute of Engineering, Tribhuvan University \\ P. O. Box 19758, Kathmandu, Nepal \\ E-mail: thapagbt@ioe.edu.np
}

\begin{abstract}
Dealing with data uncertainty in mathematical programming has been recognized as a central problem in optimization for a long time. There are two methods that have been proposed to address data uncertainty over the years, namely stochastic programming and robust optimization. In this short paper, we present the brief review of mathematical models of integer programming problem and robust optimization approach to solve them. Robust Binary optimization is also briefly presented.
\end{abstract}

Keywords: Linear programming, optimization, integer programming, robust optimization

\section{INTRODUCTION}

Mathematical programming is a broader concept representing actions that can be taken in the systems being modelled and then optimized. It maps each possible set of decisions into a single score that assures the quality of the solution as well. The optimization problems initially originated as linear programs to solve many practical problems that emerged during and after the World War II such as military logistics, diet problems and other problems in economical analysis. The linear programming problem (LPP) contains an objective function in a set of decision variables assigned with a set of constraints. The LPP model can be observed in different literatures (Thapa 2005, 2006, Bertsimas \& Tsitsiklis 1997). The LPP deals with minimization or maximization of the value of the objective function satisfying the given constraints. Each constraint requires that a linear function of the decision variables is either equal to or less than or greater than a scalar value. And each decision variable must be nonnegative.

If the set of decision variables is $x_{1}, x_{2}, \cdots, x_{n}$, general form of LPP is formulated as the following:

Minimize or maximize $\quad c_{1} x_{1}+c_{2} x_{2}+\cdots+c_{n} x_{n}$ subject to

$$
\begin{aligned}
& a_{11} x_{1}+a_{12} x_{2}+\cdots+a_{1 n} x_{n} \leq(=\text { or } \geq) b_{1} \\
& a_{21} x_{1}+a_{22} x_{2}+\cdots+a_{2 n} x_{n} \leq(=\text { or } \geq) b_{2} \\
& \begin{array}{lllll}
a_{2} x_{1}+a_{22} x_{2}+\cdots & \cdots & \ldots & \ldots
\end{array} \\
& \begin{array}{lllll}
\cdots & \ldots & \ldots & \ldots & \ldots
\end{array} \\
& a_{m 1} x_{1}+a_{m 2} x_{2}+\cdots+a_{m n} x_{n} \leq(=\text { or } \geq) b_{m}
\end{aligned}
$$

$x_{j} \geq 0, j=1,2, \cdots, n$

The coefficients $c_{j}(j=1,2, \ldots n)$ in the objective function (1) are referred as cost coefficients in minimization problems and profit coefficients in the maximization problems. The right-hand-side values $b_{1}, b_{2}, \cdots, b_{m}$ represent the amounts of available resources (especially for $\leq$ constraints) and requirements (especially for $\geq$ constraints). The coefficient values $a_{i j}$ means how much of resource or requirement $i$ is consumed or satisfied by decision $j$. This formulation is a linear program because the objective function and all the constraints are linear. The LPP formulation (1) can be expressed in its standard form as follows:

(LPP) $\quad \min c^{\prime} x$

subject to $\quad A x \leq b$

$x \geq 0$

where $c \in R^{n}, b \in R^{m}$ are given vectors and $A \in R^{m \times n}$ is a $m \times n$ matrix. If some of the decision variables are restricted to be integers, then the linear program is known to be a mixed-integer linear program. If the variables take only 0 or 1 , then the LPP is known to be $0-1$ integer programs. And if all the decision variables are restricted to be integers, then the problem is known to be integer programming problem (IPP). This problem is one of the difficult problems to solve for exact and algorithmic solutions. It arises when a large number of discrete organizational decisions have to be made under some constraints and optimization criteria. When all or 
some of the data are uncertain, then the problem becomes more difficult. One usual approach to handle this problem is stochastic programming which yields some sort of probabilistic solutions which is beyond this paper. Note that robust solution for LPP has been richly carried out in Ben-Tal and Nemirovski (1999) and BenTal and Nemirovski (2000). Convex programming approach for inexact linear programming has been dealt in Soyster (1973). Here we explain about deterministically feasible solutions for integer programs considering and redefining the data uncertainty.

\section{ROBUST INTEGER PROGRAMMING}

The new robust approach is extended to discrete optimization problems (Bertsimass \& Sim 2004). The nominal mixed integer programming (MIP) is formulated as follows:

$$
\begin{array}{ll}
\text { minimize } & c^{\prime} x \\
\text { such that } & A x \leq b \\
& x_{i} \in Z^{+}, i=1,2, \cdots, k \\
\text { where } & c \in Z^{n}, b \in Z^{m}, A \in Z^{m \times n} .
\end{array}
$$

Without loss of generality, assume that $A$ and $c$ (not $b$ ) are affected by data uncertainty.

$\operatorname{Each} a_{i j} \rightarrow \widetilde{a}_{i j} \in\left[a_{i j}-\hat{a}_{i j}, a_{i j}+\hat{a}_{i j}\right], \quad j \in N=\{1,2, \cdots, n\}$ and each $c_{j} \rightarrow \widetilde{c}_{j} \in\left[c_{j}, c_{j}+d_{j}\right]$.

Introduce $\Gamma_{i} \in\left[0,\left|J_{i}\right|\right], J_{i}=\left\{j: \hat{a}_{i j}>0\right\}$ which adjusts the robustness of the data against the level of conservatism. If less than $\Gamma_{i}$ coefficients $a_{i j}$ are subject to change, then the robust solution will deterministically be feasible. The robust MIP is formulated as follows:

$$
\text { minimize } c^{\prime} x+\max _{\left\{s_{o}::_{o} \subseteq J_{o},\left|s_{o}\right| \leq \Gamma_{o}\right\}} \sum_{j \in s_{o}} d_{j}\left|x_{j}\right|
$$

such that

$$
\begin{aligned}
& a_{i} x+\max _{\left\{s_{i}: s_{i} \subseteq J_{i}, s_{i} \mid \leq \Gamma_{i}\right\}} \sum_{j \in s_{i}} \hat{a}_{i j}\left|x_{j}\right| \leq b_{i}, \forall i \\
& x_{i} \in Z^{+}, i=1,2, \cdots, k .
\end{aligned}
$$

Theorem 1: (Bertsimas \& Weismantel, 2005) The Robust MIP (4) can be reformulated as an equivalent MIP

$$
\begin{aligned}
& \text { minimize } \quad c^{\prime} x+z_{o} \Gamma_{o}+\sum_{j \in J_{o}} p_{o j} \\
& \text { such that } \quad a_{i} x+z_{i} \Gamma_{i}+\sum_{j \in J_{i}} p_{i j} \leq b_{i}, \forall i \\
& z_{o}+p_{o j} \geq d_{j} y_{j}, \quad \forall j \in J_{o} \\
& z_{i}+p_{i j} \geq \hat{a}_{i j} y_{j}, \quad \forall i \neq 0, j \in J_{i}
\end{aligned}
$$

$$
\begin{array}{ll}
p_{i j}, z_{i}, y_{j} \geq 0 & \forall i, j \in J_{i} \\
-y_{j} \leq x_{j} \leq y_{j}, & \forall j \\
x_{i} \in Z^{+}, \quad \forall i &
\end{array}
$$

Proof: Given a vector $x^{*}$, we define $\beta_{i}\left(x^{*}\right)=\max _{\left\{s_{i}: s_{i} \subseteq J_{i}, s_{i} \mid \leq \Gamma_{i}\right\}} \sum_{j \in s_{i}} \widetilde{a}_{i j}\left|x_{j}^{*}\right|$ which equivalently is

$$
\beta_{i}\left(x^{*}\right)=\max \sum_{j \in J_{i}} \widetilde{a}_{i j}\left|x_{j}^{*}\right| z_{i j}
$$

such that

$$
\begin{aligned}
& \sum_{j \in J_{i}} z_{i j} \leq \Gamma_{i} \\
& z_{i j} \in\{0,1\}, \quad \forall i, j \in J_{i}
\end{aligned}
$$

Since the polyhedron $\left\{z: 0 \leq z_{i j} \leq 1, j \in J_{i}, \sum_{j \in J_{i}} z_{i j} \leq \Gamma_{i}\right\}$ is integral for all $\Gamma_{i} \in Z^{+}$, so $z_{i j} \in\{0,1\}$ can be replaced by $0 \leq z_{i j} \leq 1$. Now (6) becomes

$$
\beta_{i}\left(x^{*}\right)=\max \sum_{j \in J_{i}} \hat{a}_{i j}\left|x_{j}^{*}\right| z_{i j}
$$

such that

$$
\begin{aligned}
& \sum_{j \in J_{i}} z_{i j} \leq \Gamma_{i} \\
& 0 \leq z_{i j} \leq 1, \quad \forall i, j \in J_{i}
\end{aligned}
$$

The dual conversion of (7) is

$$
\beta_{i}\left(x^{*}\right)=\min \sum_{j \in J_{i}} p_{i j}+\Gamma_{i} z_{i}
$$

such that

$$
\begin{aligned}
& p_{i j}+z_{i} \geq \hat{a}_{i j}\left|x_{j}^{*}\right|, \forall j \in J_{i} \\
& p_{i j} \geq 0, \forall j \in J_{i} \\
& z_{i} \geq 0, \forall i
\end{aligned}
$$

Since (7) is feasible and bounded for all $\Gamma_{i} \in\left[0,\left|J_{i}\right|\right]$, so (8) is also feasible and bounded by strong duality with the same objective values. Using the same argument in penalty term of objective function of robust MIP (4), we get

$$
\begin{aligned}
\beta_{o}\left(x^{*}\right) & =\max _{\left\{s_{o}: s_{o} \subseteq J_{o},\left|s_{o}\right| \leq \Gamma_{o}\right\}} \sum_{j \in s_{o}} d_{j}\left|x_{j}^{*}\right| \\
& \Rightarrow \beta_{o}\left(x^{*}\right)=\max \sum_{j \in J_{o}} d_{j}\left|x_{j}^{*}\right| z_{o j}
\end{aligned}
$$

such that $\sum_{j \in J_{o}} z_{o j} \leq \Gamma_{o}$

$$
\begin{gathered}
0 \leq z_{o j} \leq 1, \quad \forall j \in J_{o} \\
\Rightarrow \quad \beta_{o}\left(x^{*}\right)=\min \sum_{j \in J_{o}} p_{o j}+\Gamma_{o} z_{o}
\end{gathered}
$$

such that $p_{o j}+z_{o} \geq d_{j}\left|x_{j}^{*}\right|$ 


$$
\begin{aligned}
& p_{o j} \geq 0, \quad \forall j \in J_{o} \\
& z_{o} \geq 0
\end{aligned}
$$

Now, putting the values of $\beta_{i}\left(x^{*}\right)$ and $\beta_{o}\left(x^{*}\right)$ in (4), we get (5). This completes the theorem.

If more than $\Gamma_{i}$ of $a_{i j}$ are subject to change, then the robust solution will be feasible with very high probability with probabilistic bound as follows:

Theorem 2: Let $x^{*}$ be optimal solution of the robust MIP, then

$$
\begin{aligned}
& P\left(\sum_{j} \widetilde{a}_{i j} x_{j}^{*}>b_{i}\right) \leq B\left(n, \Gamma_{i}\right)= \\
& \frac{1}{2^{n}}\left\{(1-\mu) \sum_{l=\lfloor v}^{n}\left(\frac{n}{l}\right)+\mu \sum_{l=\lfloor v+1}^{n}\left(\frac{n}{l}\right)\right\}
\end{aligned}
$$

where $n=\left|J_{i}\right|, \quad v=\frac{\Gamma_{i}+1}{2}$ and $\mu=v-\lfloor v\rfloor$. This bound is tight.

$$
\begin{aligned}
& \text { For } \Gamma_{i}=\theta \sqrt{n}, \lim _{n \rightarrow \infty} B\left(n, \Gamma_{i}\right)=\Phi(\theta) \\
& \text { where } \quad \Phi(\theta)=\frac{1}{\sqrt{2 \pi}} \int_{-\infty}^{\theta} \exp \left(-\frac{y^{2}}{2}\right) d y \text { is the }
\end{aligned}
$$
cumulative distribution function of a standard normal. Note that the bound on (i) is best possible but difficult to compute the sum of combination functions for large $n$. The bound on (ii) is simple to compute and very tight.

\section{ROBUST BINARY OPTIMIZATION}

The nominal case of binary optimization is formulated as

$$
\begin{aligned}
& \operatorname{minimize} c^{\prime} x \\
& \text { such that }
\end{aligned} \quad x \in\{0,1\}^{n}
$$

where feasible set $X$ is fixed, only the cost vector $\mathrm{c}$ is uncertain.

Each $c_{j} \rightarrow \widetilde{c}_{j} \in\left[c_{j}, c_{j}+d_{j}\right], \quad j \in N, d_{j} \geq 0$. The robust binary optimization is formulated as follows:

$$
Z_{R}=\min c^{\prime} x+\max _{\{s: s \subseteq N,|s| \leq \Gamma\}} \sum_{j \in s} d_{j} x_{j}
$$

such that $x \in X$.

Without loss of generality, $d_{1} \geq d_{2} \geq \cdots \geq d_{n}$ and $d_{n+1}=0$.

Theorem 3: (Bertsimas \& Sim 2003), the robust binary optimization problem can be solved by solving $n+1$ nominal problems:

$$
\begin{aligned}
& Z_{R}=\min _{l=1,2, \cdots, n+1} G_{l} \\
& \text { where } G_{l}=\Gamma d_{l}+\min \left(c^{\prime} x+\sum_{j=1}^{l}\left(d_{j}-d_{l}\right) x_{j}\right)
\end{aligned}
$$

such that $x \in X$.

Proof: Robust binary operation (10) can be written as

$$
\begin{aligned}
& Z_{R}=\min _{x \in X}\left(c^{\prime} x+\max \sum_{j \in N} d_{j} x_{j} u_{j}\right) \\
& \text { such that } \quad \sum_{j \in N} u_{j} \leq \Gamma \\
& u_{j} \in\{0,1\}, \quad j \in N .
\end{aligned}
$$

Since the polyhedron $\left\{u: 0 \leq u_{j} \leq 1, j \in N, \sum_{j \in N} u_{j} \leq \Gamma\right\}$ is integral for all $\Gamma \in Z^{+}$, so $u_{j} \in\{0,1\}$ can be replaced by $0 \leq u_{j} \leq 1$. Therefore (12) becomes

such that

$$
Z_{R}=\min _{x \in X}\left(c^{\prime} x+\max \sum_{j \in N} d_{j} x_{j} u_{j}\right)
$$

$$
\begin{aligned}
& \sum_{j \in N} u_{j} \leq \Gamma \\
& 0 \leq u_{j} \leq 1, \quad j \in N .
\end{aligned}
$$

Taking dual of inner maximization problem and applying strong duality, we have

$$
Z_{R}=\min _{x \in X} c^{\prime} x+\min \left(\Gamma \theta+\sum_{j \in N} y_{j}\right)
$$

such that $y_{j}+\theta \geq d_{j} x_{j}$

$$
\begin{aligned}
& y_{j}, \theta \geq 0, \quad j \in N \\
\Rightarrow \quad & Z_{R}=\min \left(c^{\prime} x+\Gamma \theta+\sum_{j \in N} y_{j}\right)
\end{aligned}
$$

such that

$$
\begin{aligned}
& y_{j}+\theta \geq d_{j} x_{j} \\
& x \in X, \quad y_{j}, \theta \geq 0 .
\end{aligned}
$$

Clearly, an optimal solution $\left(x^{*}, y^{*}, \theta^{*}\right)$ of (13) satisfies $y_{j}^{*}=\max \left(d_{j} x_{j}^{*}-\theta^{*}, 0\right)$.

Therefore (13) becomes

$Z_{R}=\min _{x \in X, \theta \geq 0}\left(\Gamma \theta+c^{\prime} x+\sum_{j \in N} \max \left(d_{j} x_{j}-\theta, 0\right)\right)$

i.e., $Z_{R}=\min _{x \in X, \theta \geq 0}\left(\Gamma \theta+c^{\prime} x+\sum_{j \in N} \max \left(d_{j}-\theta, 0\right) x_{j}\right)$,

since $X \subseteq\{0,1\}$.

To find optimal value for $\theta$, we decompose the set of positive real numbers $R^{+}$in the intervals

$\left[0, d_{n}\right],\left[d_{n}, d_{n-1}\right], \cdots,\left[d_{2}, d_{1}\right],\left[d_{1}, \infty\right]$ and we get

$\sum_{j \in N} \max \left(d_{j}-\theta, 0\right) x_{j}= \begin{cases}\sum_{j=1}^{l-1}\left(d_{j}-\theta\right) x_{j}, & \text { if } \theta \in\left[d_{1}, d_{l-1}\right], l=n+1, \cdots, 2 \\ 0 & \text { if } \theta \in\left[d_{1}, \infty\right)\end{cases}$

Therefore, $Z_{R}=\min _{l=1,2, \cdots, n+1} Z_{l}$

where $Z_{l}=\min _{x \in X, \theta \in\left[d_{l}, d_{l-1}\right]}\left(\Gamma \theta+c^{\prime} x+\sum_{j=1}^{l-1}\left(d_{j}-\theta\right) x_{j}\right)$ 
Since we are optimizing a linear function of $\theta$ over the intervals $\left[d_{l}, d_{l-1}\right]$, the optimal solution is obtained for $\theta=d_{l}$ or $\theta=d_{l-1}$, and thus for $l=1,2, \cdots, n+1$, we have $Z_{l}=\min \left(\Gamma d_{l}+\min _{x \in X}\left(c^{\prime} x+\sum_{j=1}^{l-1}\left(d_{j}-d_{l}\right) x_{j}\right), \Gamma d_{l-1}+\min _{x \in X}\left(c^{\prime} x+\sum_{j=1}^{l-1}\left(d_{j}-d_{l-1}\right) x_{j}\right)\right)$ i.e.,

$Z_{l}=\min \left(\Gamma d_{l}+\min _{x \in X}\left(c^{\prime} x+\sum_{j=1}^{l}\left(d_{j}-d_{l}\right) x_{j}\right), \Gamma d_{l-1}+\min _{x \in X}\left(c^{\prime} x+\sum_{j=1}^{l-1}\left(d_{j}-d_{l-1}\right) x_{j}\right)\right)$ Now (14) becomes

$Z_{R}=\min \left(\Gamma d_{1}+\min _{x \in X} c^{\prime} x, \cdots \cdots, \Gamma d_{l}+\min _{x \in X}\left(c^{\prime} x+\sum_{j=1}^{l}\left(d_{j}-d_{l}\right) x_{j}\right), \cdots, \min _{x \in X}\left(c^{\prime} x+\sum_{j=1}^{n} d_{j} x_{j}\right)\right)$ This completes the theorem.

For further detail of robust discrete optimization and network flows, we refer to Bertsimas and Sim (2003) and Bertsimas and Weismantel (2005).

\section{CONCLUSIONS}

Mathematical programming has a very wide and important role in real life problems. There are two types of optimization problems, namely continuous optimization and discrete optimization. Data uncertainty is obvious in any case of the optimization. Therefore the only deterministic solution cannot address the data uncertainty and sometimes the solution does not guarantee the exactness. To address the uncertainty in data, there are two approaches, the first is stochastic programming and the other is robust programming. The robust optimization defines the robustness of uncertain data taking some intervals and handle the problems deterministically. The general case of integer programming is NP-hard problem and the existence of exact solution is not possible. However, there is always a possibility of finding near to optimal solution, for example heuristic approaches.

\section{REFERENCES}

Ben-Tal, A. and Nemirovski, A. 1999. Robust Solutions to Uncertain Programs. Operations Research Letters 25: 1-13.

Ben-Tal, A. and Nemirovski, A. 2000. Robust solutions of Linear Programming Problems Contaminated with Uncertain Data. Mathematical Programming 88: 411-424.

Bertsimas, D. and Sim, M. 2003. Robust discrete optimization and network flows. Mathematical Programming 98: 49-71.

Bertsimas, D. and Sim, M. 2004. The Price of Robustness. Operations Research 52(1): 35-53.

Bertsimas, D and Weismantel, R. 2005. Optimization Over Integers. Dynamic Ideas, Massachusetts, USA. ISBN: 0-9759146-2-6.

Bertsimas, D and Tsitsiklis, J.N. 1997. Introduction to Linear Optimization. Massachusetts Institute of Technology, Athena Scientific, Belmont, Massachusetts, USA.

Soyster, A.L. 1973. Convex Programming with setinclusive Constraints and Applications to inexact linear programming. Operations Research 21: 1154-1157.

Thapa, G.B. 2005. Basic Concepts on Integer Optimization. Central Dept. of Mathematics, Tribhuvan University, Nepal. The Proceedings of The Two days Seminar on Application of Mathematics 3: 33-43.

Thapa, G.B. 2006. Computational Complexity and Integer Programming. Mathematical Sciences and Applications 60-70, Kathmandu University, Nepal. 